\title{
Refractive index sensing in an all-solid twin-core photonic bandgap fiber
}

\author{
Yuan, Scott Wu; Town, Graham E.; Bang, Ole
}

Published in:

I E E E Sensors Journal

Link to article, DOI:

10.1109/JSEN.2010.2040174

Publication date:

2010

Document Version

Publisher's PDF, also known as Version of record

Link back to DTU Orbit

Citation $(A P A)$ :

Yuan, S. W., Town, G. E., \& Bang, O. (2010). Refractive index sensing in an all-solid twin-core photonic bandgap fiber. I E E E Sensors Journal, 10(7), 1192-1199. https://doi.org/10.1109/JSEN.2010.2040174

\section{General rights}

Copyright and moral rights for the publications made accessible in the public portal are retained by the authors and/or other copyright owners and it is a condition of accessing publications that users recognise and abide by the legal requirements associated with these rights.

- Users may download and print one copy of any publication from the public portal for the purpose of private study or research.

- You may not further distribute the material or use it for any profit-making activity or commercial gain

- You may freely distribute the URL identifying the publication in the public portal 


\title{
Refractive Index Sensing in an All-Solid Twin-Core Photonic Bandgap Fiber
}

\author{
Wu Yuan, Graham E. Town, Senior Member, IEEE, and Ole Bang
}

\begin{abstract}
We describe a highly sensitive refractive index sensor based on a twin-core coupler in an all-solid photonic bandgap guiding optical fiber. A single hole acts as a microfluidic channel for the analyte, which modifies the coupling between the cores, and avoids the need for selective filling. By operating in the bandgap guiding regime the proposed sensor is capable of measuring refractive indices around that of water, and because the analyte varies the coupling coefficient (i.e., instead of phase matching condition) the device is capable of both high sensitivity and a relatively large dynamic range.
\end{abstract}

Index Terms-Directional coupler, fiber sensor, photonic bandgap fiber, refractive index sensor.

\section{INTRODUCTION}

$\mathbf{F}$ IBER-OPTIC biosensors have the potential for providing simple, rapid, and continuous in situ monitoring of biomolecules in the biomedical, pharmaceutical, environmental, defense, bioprocessing, and food technology areas [1]. Low-loss delivery of laser light, long interaction lengths, low fabrication costs, and the ability to both excite target molecules and capture their emitted light, are important advantages of optical fibers in the context of biosensing. Despite their geometry, optical fibers may also be incorporated into disposable lab-on-a-chip biosensors as the key sensing element [2]. The main goal is to develop fiber-optic biosensors capable of performing rapid and reliable selective immunoassays on unlabeled samples [3].

An immense variety of configurations and working principles have been demonstrated in optical fiber biosensors (e.g., see [1] and [3] for recent reviews). Flourescence-based fiber-optical biosensors may be formally label-free by having the label molecules attached to the probe instead of the sample, such as in the RAPTOR [4]. However, typical label-free fiber-optic biosensors depend upon the change of a resonance (grating period, surface plasmon, Fabry-Perot cavity length, phase matching condition, etc.) introduced by the presence of a

Manuscript received August 03, 2009; revised December 18, 2009; accepted December 29, 2009. Date of current version May 19, 2010. This work was supported in part by the Danish Agency for Science Technology and Innovation, in part by the Australian Research Council, and in part by the Danish National Advanced Technology Foundation. The associate editor coordinating the review of this paper and approving it for publication was Prof. Miguel Andres.

W. Yuan and O. Bang are with DTU Fotonik, Department of Photonics Engineering, Technical University of Denmark, DK-2800 Kgs. Lyngby, Denmark (e-mail: wyuan@fotonik.dtu.dk; oban@fotonik.dtu.dk).

G. E. Town is with MQ Photonics, Department of Physics and Engineering, Macquarie University, Macquarie NSW 2109, Australia (e-mail: gtown@elec.mq.edu.au).

Digital Object Identifier 10.1109/JSEN.2010.2040174 biological agent [3], [5]-[9]. Such sensors are truly label-free in that they require no labeled molecules, thereby simplifying the biochemistry.

Microstructured optical fiber (MOF) has an array of holes running along the entire length of the fiber [10], [11] and consequently has a number of advantages for sensing applications. First, the holes in the structure can be filled with analyte, allowing biochemical reactions and sensing regions to be located inside the fiber, in close proximity to the guided light. Biological samples may therefore be probed by the optical field without removing the fiber coating and cladding, thus maintaining the robustness of the fiber. Furthermore, the holes in a MOF are typically small and thus only minute sample volumes are required (picoliter regime) to achieve high sensitivity.

The optical properties of MOFs are primarily determined by the position, size, and shape of the holes, and they have a number of unique characteristics compared to conventional step-index fibers. In particular, MOFs with a periodic microstructure (i.e., photonic crystal fibers) can be designed to guide light in a low index region (e.g., air hole) by the photonic bandgap (PBG) effect [12], [13]. All-solid PBG MOFs have also been demonstrated [14]-[17].

MOFs therefore provide a flexible platform for optical sensing of a wide range of analyte refractive index in a variety of configurations. Two general configurations for interaction between the light and analyte in MOFs may be identified: i) the analyte may be located in the evanescent field of the waveguide, as first proposed by Monro et al. [18], [19] or ii) the analyte may act as a waveguide, providing maximal overlap with the optical field, e.g., [20] and [21]. Furthermore the analyte index may be either: a) higher or b) lower than the refractive index of the fiber host material (i.e., refractive index guiding, or photonic bandgap guiding, respectively).

Additionally, multicore waveguide structures are readily realized in MOFs [22], enabling configurations in which the analyte modifies the coupling coefficient (i.e., as distinct from the phase matching, as previously demonstrated) between two or more waveguides. Couplers in microstructured fibers have been well studied [23]-[32], and arrays of waveguides coupled by the analyte have been shown to be capable of providing enhanced sensitivity for refractive index sensing [33], [34]. In this work, we demonstrate the potential of such structures for realizing simple and sensitive biosensors in the context of a twin-core photonic bandgap guiding MOF.

Our main interest is in polymer microstructured optical fibers, which can be fabricated with a wealth of different hole-structures [35], [36]. Bandgap guiding fibers have also 
been manufactured in polymer [37]. Importantly, polymer is less brittle and much easier to functionalize than silica, and hence polymer MOFs are usually preferred for biosensing applications [38], [39]. Polymer microstructured fibers have most commonly been fabricated in polymethylmethacrylate (PMMA), nevertheless other polymers also show promise; for example, TOPAS polymer MOFs have been fabricated and shown to have advantageous properties for both fiber drawing and biosensing [40], [41] and for guiding in the terahertz regime [42]. Polymers are also available over a relatively wide range of refractive index, from $\mathrm{n}=1.34$ in fluorinated polymers to $\mathrm{n}=1.58$ or more for polycarbonate, etc.

Although many MOF sensors have been demonstrated experimentally, they have mostly not been in the context of label-free biosensing. Furthermore, sensing in aqueous media has usually been restricted to evanescent wave sensing configurations in which there is poor overlap between the optical field and analyte, which severely limits sensitivity. However, a prerequisite for a label-free biosensor to function, is that it is able to work as a sensitive refractive index sensor, and thus the two types of sensors are often discussed in parallel. The best sensitivities of label-free MOF biosensors to date have been reported in devices in which the analyte modifies the phase matching, or peak coupling wavelength, between coupled modes. For example, Rindorf et al. demonstrated a sensitivity of $1.4 \mathrm{~nm}$ shift of a long-period grating resonance per $\mathrm{nm}$ biolayer $(1.4 \mathrm{~nm} / \mathrm{nm})$ [5], and Ott et al. [6] predicted a sensitivity of $10.4 \mathrm{~nm} / \mathrm{nm}$ in a four-wave mixing-based label-free biosensor. Wu et al. [9] recently demonstrated a sensitivity of $30100 \mathrm{~nm}$ per refractive index unit (nm/RIU) in a refractive index guiding twin-core silica MOF operating just above cutoff of the selectively filled analyte channel, shown schematically in Fig. 1(a). However, the latter device had a limited dynamic range, $\Delta \mathrm{n}=0.022$, and is not suitable for sensing refractive indices less than that of the fiber host. It has now been shown that coating the holes and using flourinated polymer MOFs will allow to extend the regime of operation to low indices, such as water [43].

In this paper, we describe an improved MOF refractive index sensor configuration using a balanced directional coupler in an all-solid photonic bandgap guiding (PBG) twin-core MOF. The analyte channel sits between two solid PBG cores, as illustrated in Fig. 1(b). Since only the analyte channel is empty, no selective filling is required, i.e., the sensor could be loaded simply by dipping into the sample. We analyze the sensitivity of the twin-core PBG MOF sensor with specified lengths in two modes of operation: i) transmission of a single wavelength source, with the detected intensity determined by the analyte refractive index and ii) broadband sensing of the shift in peaks and/or nulls of the coupled wavelengths with analyte refractive index. We show that the sensor operation can best be understood in terms of interference between the odd and even supermodes of the coupled waveguides and can, in theory, provide enhanced sensitivity compared with sensing devices which rely on a shift of resonance or phase matching. We also identify some unique features of the coupled waveguide sensor, including scalability of the sensitivity to index changes with sensor length, and the potential for refractive index sensing over a wide dynamic range. (a)

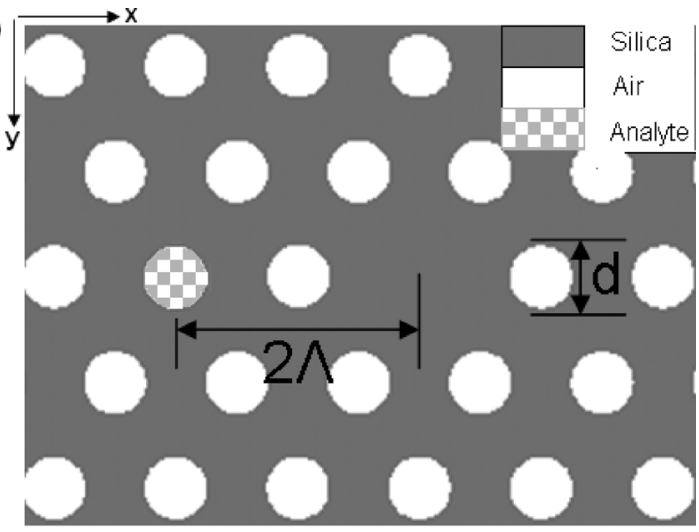

(b)

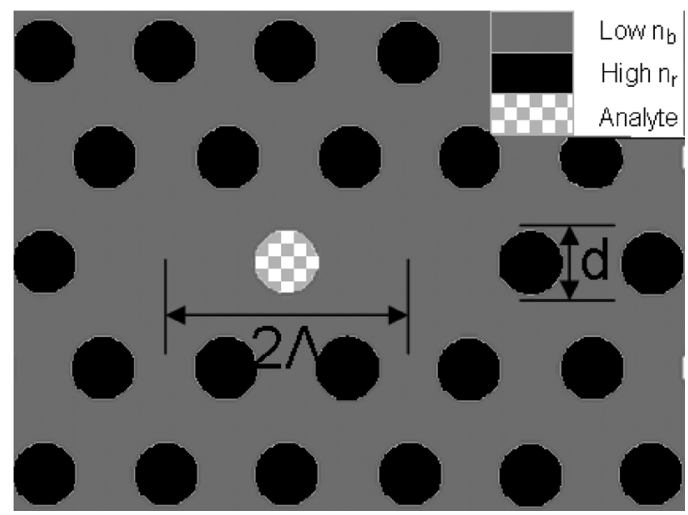

Fig. 1. Triangular microstructure with pitch $\Lambda$ and hole diameter $d$ of (a) the twin-core index-guiding MOF studied in [9] and (b) our proposed solid twincore PBG sensor, fabricated from a host material with low index $\mathrm{n}_{\mathrm{b}}$ and rod inclusions with higher index $n_{r}$.

\section{PRINCIPLE AND ModELING}

\section{A. Sensor Description}

As shown in Fig. 1(b), the proposed PBG MOF sensor has a single microfluidic analyte channel (refractive index $n_{a}$ ) between two solid and identical low index cores. The two cores are separated by two times the pitch, i.e., $2 \Lambda$, and the cladding is a triangular array of high-index rods of diameter $\mathrm{d}=0.53 \Lambda$, separated by a pitch of $\Lambda=3.2 \mu \mathrm{m}$. The fiber is designed to be fabricated using two different polymer materials, i.e., Poly 1 and Poly2, where Poly1 is the low refractive index background material $\left(n_{\mathrm{b}}=1.34\right)$, and Poly2 is used for the high index rods $\left(n_{\mathrm{r}}=1.53\right)$. We neglect material dispersion, which is not relevant if using a single wavelength source.

\section{B. Principle of Operation}

Each core in the PBG MOF has broad transmission windows, or bandgaps, in which optical guidance occurs. The bandgap wavelengths and bandwidths are determined by the periodicity, geometry, and refractive indices of the high index inclusions relative to the low index host. The bandgaps for the fiber described in this work were calculated by using MIT's plane-wave package [44] and are shown in Fig. 2(a).

The cores of the PBG MOF form a balanced directional coupler. Coupling between the two cores occurs due to interaction via their evanescent fields, causing a periodic transfer of optical power from one core to the other and back. The coupling can also be understood and analyzed in terms of a pair 



Fig. 2. (a) Photonic bandgaps of one of the cores of the PGB fiber in isolation, shown as effective index $\mathrm{n}_{\text {eff }}$ versus normalized frequency $\lambda / \Lambda$, for $\mathrm{n}_{\mathrm{b}}=1.34$ and $\mathrm{n}_{\mathrm{r}}=1.53$. The refractive index of the cores is shown with a solid black line. (b) Effective indices of the $\mathrm{x}$ - and $\mathrm{y}$-polarized even and odd supermodes in the second bandgap. $\Lambda=3.2 \mu \mathrm{m}, \mathrm{d}=0.53 \Lambda$.

of supermodes, i.e., a symmetric (even) supermode and an antisymmetric (odd) supermode. The effective indices $n_{\text {eff }}$ and field distributions of the even and odd supermodes of the dual-core PBG MOF were calculated using the fully vectorial finite element package COMSOL ${ }^{\mathrm{TM}}$. The effective indices in the second bandgap are shown in Fig. 2(b), from which we see that the difference between the $x$ - and $y$-polarized supermodes is small. In the following, we therefore use as effective index the average effective indices of the two orthogonal polarizations, $n_{\mathrm{eff}}=$ $\left(n_{x}+n_{y}\right) / 2$.

For a balanced coupler the proportion of the launched power, $P_{1}(0)$, coupled to the other core in a device of length $L$ is

$$
T_{21}=\frac{P_{2}(L)}{P_{1}(0)}=\sin ^{2}\left(\frac{\theta}{2}\right)
$$

where $\theta$ is the phase difference between the two supermodes, defined by

$$
\theta=k \cdot L \cdot\left|n_{\text {odd }}-n_{\text {even }}\right|=k \cdot L \cdot \Delta n_{\text {eff }}\left(\lambda, n_{a}\right) .
$$

(a)

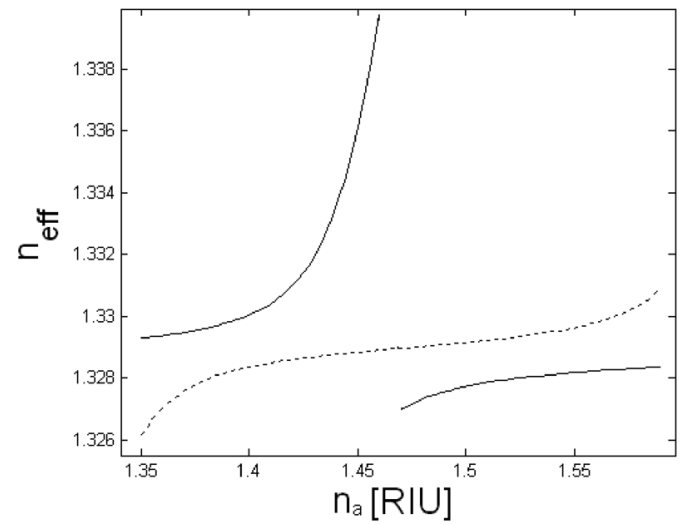

(b)

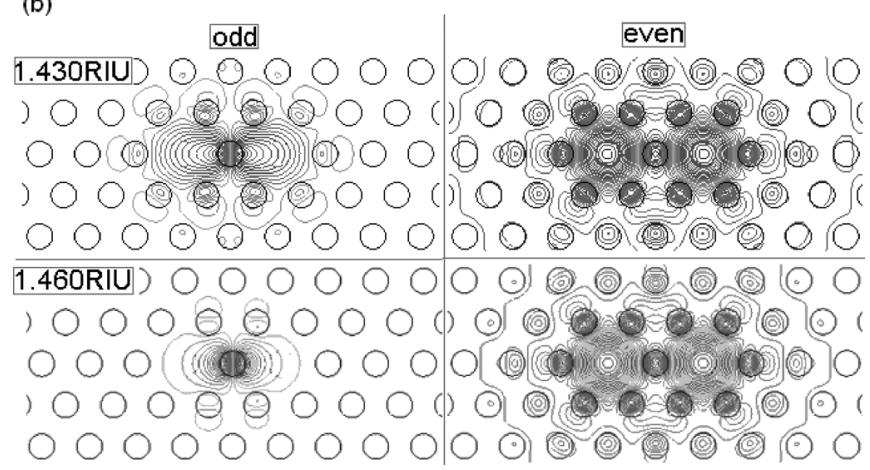

Fig. 3. (a) Effective indices of the odd supermode ( $n_{\text {odd }}$, solid line), and of the even supermode ( $n_{\text {even }}$, dashed line) versus the analyte index, $n_{a}$, at wavelength $1250 \mathrm{~nm}$. (b) Representative electric field contours of the odd and even supermodes in the twin-core PBG MOF at $n_{a}=1.430$ and $1.460, \Lambda=3.2 \mu \mathrm{m}$, $\mathrm{d}=0.53 \Lambda$.

In (1) and (2), $L$ is the length of the coupler, $k=2 \pi / \lambda$ is the free space propagation constant, $\lambda$ is the free space wavelength, and $\mathrm{n}_{\text {odd }}$ and $\mathrm{n}_{\text {even }}$ are the effective indices of the even and odd supermodes, respectively, and $\Delta n_{\text {eff }}=\left|n_{\text {odd }}-n_{\text {even }}\right|$.

The coupling length, $L_{c}$, is defined as the distance at which the optical input has transferred completely from one core to the other, i.e., at $\theta=\pi$, so

$$
L_{c}=\frac{\lambda}{2 \cdot \Delta n_{\mathrm{eff}}\left(\lambda, n_{a}\right)} .
$$

The coupling length is inversely proportional to the coupling coefficient between the two cores, and varies rapidly with confinement of the core modes and separation between the cores, $2 \Lambda$, and in the twin-core PBG MOF will also depend upon the refractive index of the analyte, $n_{a}$.

In conventional twin-core index-guiding MOFs, $L_{c}$ increases monotonically with increasing frequency [22]-[27]. Couplers in photonic bandgap guiding fibers behave similarly for low frequencies inside the bandgap, however, the coupling length does not always increase monotonically with frequency, and can be modified by coupling to high-index inclusions [28], [31]. A microanalyte channel placed between two bandgap guiding cores can therefore be expected to have a strong influence on the coupling between the cores, and hence the transmittance of the coupler at any given wavelength.

The strong influence of the analyte channel on coupling and transmittance may also be understood in terms of the effect on the coupler's odd and even supermodes, as shown in Fig. 3. 
Fig. 3(a) indicates that the average effective index of the odd supermode (solid line) is considerably more sensitive to variation of the analyte refractive index than the even supermode (dashed line). The reason is illustrated in Fig. 3(b), where it can be seen that the odd supermode is concentrated in and around the analyte channel whereas the even supermode has minimal overlap with the analyte. Consequently, even though the launched optical field does not overlap significantly with the analyte, the large differential overlap of the supermodes with the analyte results in enhanced sensitivity compared to other evanescent field sensors, a result of the coupler's symmetry.

Fig. 3(a) also shows that at a specific analyte index (in this case $n_{a} \sim 1.46$ ) the ordering of the supermodes' effective indices is reversed from $n_{\text {odd }}>n_{\text {even }}$ to $n_{\text {odd }}<n_{\text {even }}$. This is because at this analyte index the odd supermode couples with the second-order refractive index guiding mode of the analyte channel.

In the following sections, we analyze the performance of the bandgap coupler sensor using two possible methods of interrogation. The first and simplest method is based upon measurement of sensor transmittance at a single wavelength. The second method requires a broadband optical source and spectrum analyzer to measure the sensor's transmittance as a function of wavelength. In both cases, we assume the sensor length and/or transmittance have been calibrated in air before introduction of the analyte. Note that the results shown in this paper are for guidance and coupling in the second bandgap of the fiber, as this is expected to result in higher sensitivity than operation in the 1st bandgap, i.e., due to the faster change in mode confinement near the edges of the second bandgap, while avoiding fabrication difficulties and losses associated with higher order bandgaps. Furthermore, due to the high index contrast, there should be no problem with confinement losses as there would be in a low index contrast all-solid PBG fiber [15].

\section{Transmittance Sensing Scheme}

If light at a specified wavelength is incident on one of the cores of a twin-core PBG MOF, the normalized intensity leaving the opposite core after propagating through a twin-core PBG MOF sensor with length $L$ is given by (1). Consequently changes in refractive index may be determined from the variation in transmitted intensity at a fixed wavelength, $\lambda$, as shown in Fig. 4 for a $1 \mathrm{~mm}$ long sensor (i.e., equal to a single coupling length at $1100 \mathrm{~nm}$ with $n_{a}=1.4$ ).

Note that as the analyte refractive index and coupling increase, the transmittance passes through a series of nulls and peaks as the coupling length reduces. The periodic variation of transmittance is as expected from (1), however, the fact that the period itself is reducing indicates that the coupling between the PBG cores of the MOF changes increasingly rapidly with increasing index in the analyte channel. The rapid change of coupling length with analyte index, especially near the short wavelength edge of the bandgap, is illustrated in Fig. 5(a) and (b).

The varying dependence of coupling on analyte index, allows the sensor to be designed for maximum dynamic range or for maximum sensitivity to index change. Figs. 4 and 5 show how this design tradeoff may be implemented simply in practice by an appropriate choice of source wavelength. For example, if wishing to measure refractive index around $n_{a}=1.425$ with

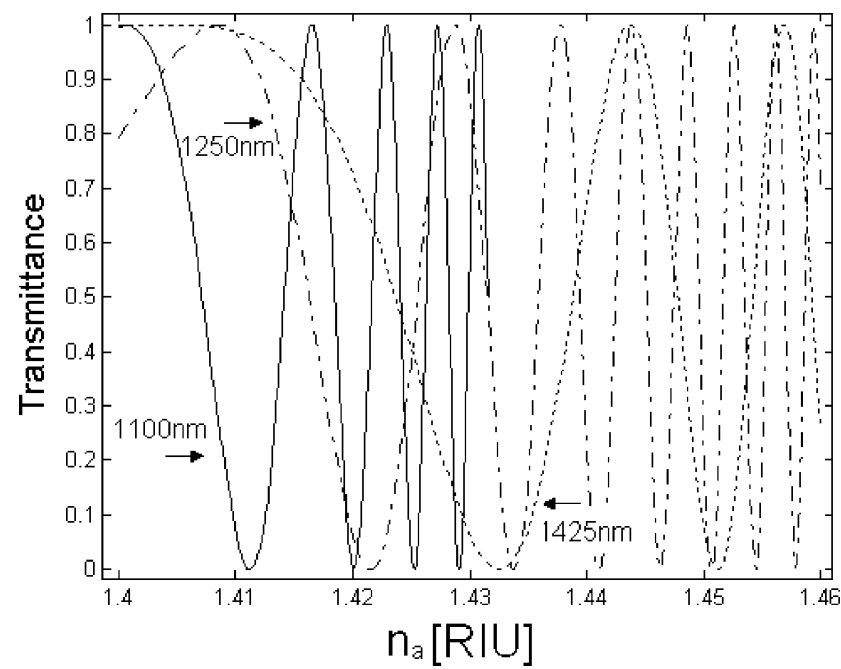

Fig. 4. Transmittance to the opposite core from the source or launch core as a function of analyte index in a $1 \mathrm{~mm}$ long PBG MOF sensor as described in the text. The transmittance characteristic is plotted for 3 wavelengths; $1100 \mathrm{~nm}$ (solid), $1250 \mathrm{~nm}$ (dash-dotted), and $1425 \mathrm{~nm}$ (dotted). $\Lambda=3.2 \mu \mathrm{m}, \mathrm{d}=0.53 \Lambda$.



(b)

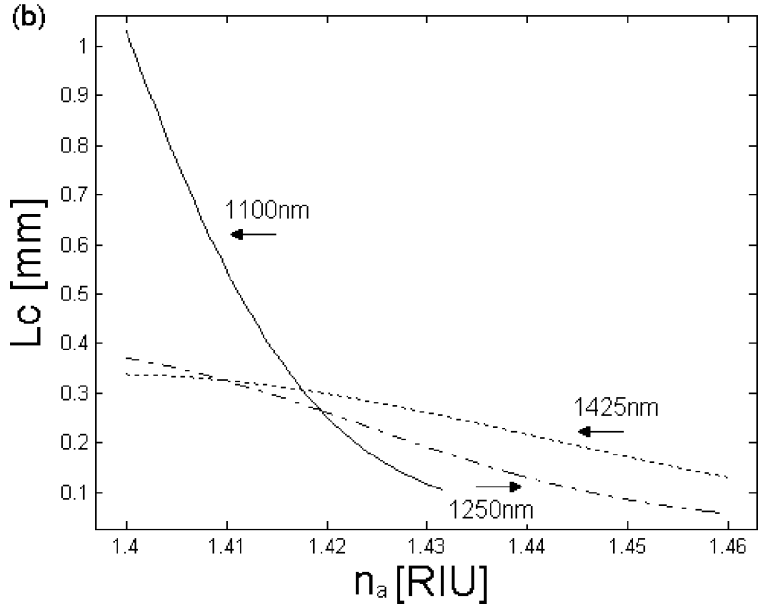

Fig. 5. Coupling length $\mathrm{L}_{c}$, after which all power is coupled from one core to the other in the twin-core PBG fiber, operated in the second bandgap. (a) Shows $\mathrm{L}_{c}$ versus the wavelength for a fixed analyte refractive index of $\mathbf{n}_{a}=1.4$ (solid curve) and $\mathrm{n}_{a}=1.401$ (dotted curve). (b) Shows the dependence on the analyte refractive index $\mathbf{n}_{a}$ for a fixed wavelength of $1100 \mathrm{~nm}$ (solid), $1250 \mathrm{~nm}$ (dashdotted), and $1425 \mathrm{~nm}$ (dotted). $\Lambda=3.2 \mu \mathrm{m}, \mathrm{d}=0.53 \Lambda$.

high sensitivity, one would use an $1100 \mathrm{~nm}$ source which provides a much more rapid change in transmittance with analyte index than longer wavelengths, such as at $1425 \mathrm{~nm}$, which 



Fig. 6. Difference in effective index of the odd and even supermodes, $\Delta \mathbf{n}_{\text {eff }}$, of the twin-core PBG fiber operated in the second bandgap. (a) Shows $\Delta \mathrm{n}_{\text {eff }}$ versus the wavelength for a fixed analyte refractive index of $\mathbf{n}_{a}=1.4$ (solid curve) and $\mathbf{n}_{a}=1.401$ (dotted curve). (b) Shows the dependence on the analyte refractive index $\mathrm{n}_{a}$ for a fixed wavelength of $1100 \mathrm{~nm}$ (solid), $1250 \mathrm{~nm}$ (dashdotted), and $1425 \mathrm{~nm}$ (dotted). $\Lambda=3.2 \mu \mathrm{m}, \mathrm{d}=0.53 \Lambda$.

would be used instead to provide a larger dynamic range. If one was able to determine the number of coupling lengths in the sensor, e.g., by determining the slope of the transmission characteristic at 50\% coupling, then one could measure the analyte index with both high sensitivity and wide dynamic range.

For best sensitivity the sensor must be biased to operate at $50 \%$ transmittance, e.g., with $\theta=\pi / 2$, or $L=L_{c} / 2$ (or an odd multiple thereof). In practice this condition may be achieved by fabricating the device length to be an odd number of half-coupling lengths, or by temperature or wavelength tuning. Under these conditions the sensitivity $\partial T_{21} / \partial n_{a}$ of the coupler-based sensor will be

$$
\begin{aligned}
\frac{\partial T_{21}}{\partial n_{a}} & = \pm\left(N+\frac{1}{2}\right) \cdot k \cdot L_{c} \cdot \frac{\partial\left(\Delta n_{\mathrm{eff}}\right)}{\partial n_{a}} \\
& = \pm\left(N+\frac{1}{2}\right) \cdot \frac{\pi}{\Delta n_{\mathrm{eff}}} \cdot \frac{\partial\left(\Delta n_{\mathrm{eff}}\right)}{\partial n_{a}}
\end{aligned}
$$

which depends directly on $\partial\left(\Delta n_{\mathrm{eff}}\right) / \partial n_{a}$ and scales with device length or number of coupling lengths, $\mathrm{N}$.

Numerical calculations of $\Delta n_{\text {eff }}$ versus $n_{a}$, shown in Fig. 6, confirm that the maximum sensitivity is obtained by operating

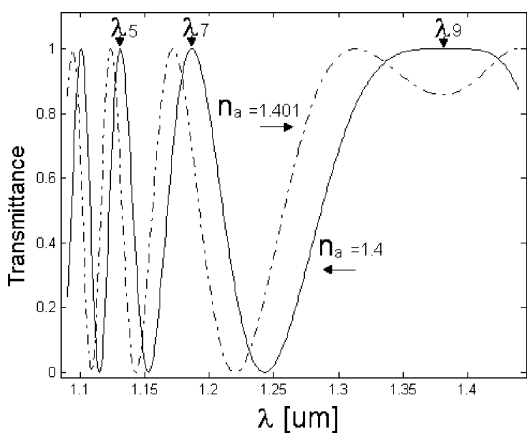

Fig. 7. Transmittance dependence on the wavelength $\lambda$ for a fixed fiber length $\mathrm{L}=3 \mathrm{~mm}$ and a fixed analyte refractive index of $\mathrm{n}_{a}=1.4$ (solid curve) and $\mathrm{n}_{a}=1.401$ (dash-dotted curve). At the indicated wavelength $\lambda_{\mathrm{N}}$ the fiber length $\mathrm{L}$ is $\mathrm{N}$ times the coupling length $\mathrm{L}_{c} . \Lambda=3.2 \mu \mathrm{m}, \mathrm{d}=0.53 \Lambda$.

near the short wavelength edge of the bandgap. Taking the previous example of measuring analyte index around $n_{a}=1.425$ in a $1 \mathrm{~mm}$ long coupler, then using a $1425 \mathrm{~nm}$ source would give maximum sensitivity $\partial T_{21} / \partial n_{a}=7091.6 \% / \mathrm{RIU}$, and a dynamic range of $\Delta \mathrm{n}_{a} \sim 0.02 \mathrm{RIU}$. Using an $1100 \mathrm{~nm}$ source, the maximum sensitivity would be $65872.9 \% / \mathrm{RIU}$, and the dynamic range approximately only $0.001 \mathrm{RIU}$. Hence an order of magnitude change in sensitivity and dynamic range may be obtained by appropriate choice of wavelength. Also, it should be noted that the sensor analyzed here is relatively short $(1 \mathrm{~mm})$, however, because the sensitivity scales with length (though with a proportional reduction in dynamic range), significantly higher sensitivities would be quite achievable.

\section{Spectral Sensing Scheme}

The sensor's transmittance at all wavelengths can be measured using a broadband optical source and spectrum analyzer to gain additional information (e.g., regarding analyte dispersion, analyte channel length, etc.). This approach also lends itself to novel interpretations of the data.

For example, when launching the light into one core of a $3 \mathrm{~mm}$ long sensor and monitoring the output from the opposite core, a periodic variation of transmittance with wavelength is evident, in which the peaks and nulls shift with changes in $n_{a}$, as illustrated in Fig. 7. All light coupled into one core is transferred to the other core at wavelengths corresponding to an odd number of coupling lengths, $L=(2 N+1) L_{c}$. The wavelength(s) at which complete coupling occurs will be denoted $\lambda_{\mathrm{N}}$, and referred to as the "coupling wavelength(s)." Any variation of $n_{a}$ will cause a change in the phase mismatch condition and therefore a shift in the coupling wavelengths.

Fig. 8 shows the shift of the coupling wavelength(s) with the variation of analyte index for the $3 \mathrm{~mm}$ long PBG MOF described previously, in which the coupling length for $\lambda_{9}$ is $L_{c}=$ $0.334 \mathrm{~mm}$ at $\mathrm{n}_{a}=1.4$. The sensitivity of the spectral sensing scheme may be defined as the derivative of $\lambda_{\mathrm{N}}$ with respect to the analyte refractive index $n_{a}$, and is shown in Fig. 9(a). The largest wavelength shift and hence greatest sensitivity occurs at the long wavelength side of the bandgap, in this case $\lambda_{9}$. In this example, tracking the shift of the coupling wavelength $\lambda_{9}$ provides a sensitivity of over $10^{5} \mathrm{~nm} /$ RIU in the $3 \mathrm{~mm}$ long fiber. Fig. 9(b) shows the sensitivity of the spectral sensing scheme as 


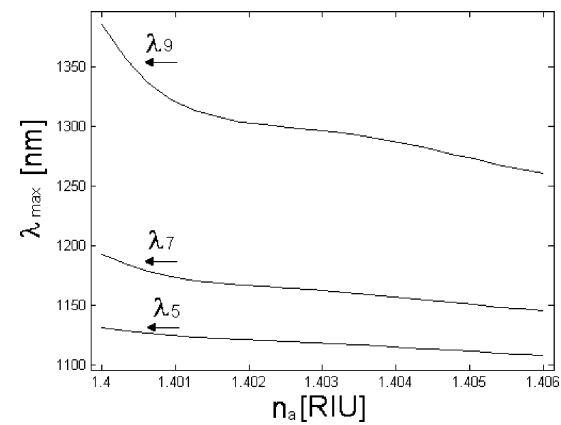

Fig. 8. Dependence of $\lambda_{N}$ on the analyte refractive index $n_{a}$ for a device length of $\mathrm{L}=3 \mathrm{~mm}$. At $\lambda_{\mathrm{N}}$ the device length $\mathrm{L}$ is $\mathrm{N}$ times the coupling length $L_{c}$ of the solid twin-core PBG fiber. Thus, if the sensor is optically excited in the right core then at $\lambda_{\mathrm{N}}$ all the power is transferred to the left core. $\Lambda=3.2 \mu \mathrm{m}$, $\mathrm{d}=0.53 \Lambda$.
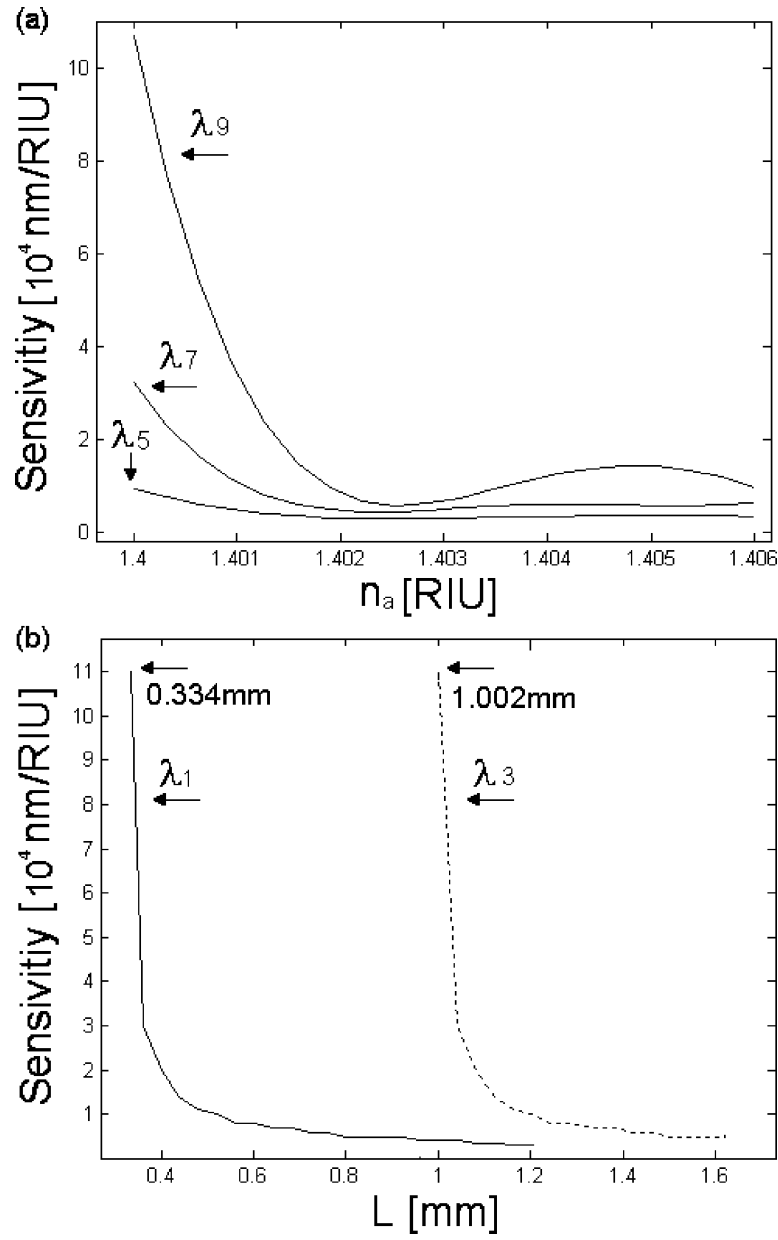

Fig. 9. Sensitivity obtained by tracking the coupling wavelength $\lambda_{N}$, and defined as the derivative of $\lambda_{\mathrm{N}}$ with respect to the analyte refractive index $n_{a}$. (a) Sensitivity versus $n_{a}$ with a fixed device length of $L=3 \mathrm{~mm}$ for different tracking wavelengths. (b) Sensitivity versus device length with $n_{a}=1.4$ for different tracking wavelengths. $\Lambda=3.2 \mu \mathrm{m}, \mathrm{d}=0.53 \Lambda$.

a function of fiber length, and shows that the highest sensitivity is obtained when the sensor length approaches on odd number of coupling lengths of the tracked wavelength.

From (3), the coupling wavelength, $\lambda_{\mathrm{N}}$, is defined by

$$
\lambda_{\mathrm{N}}=2 \cdot N \cdot L_{c} \cdot \Delta n_{\mathrm{eff}}\left(\lambda_{\mathrm{N}}, n_{a}\right)
$$

and therefore the sensitivity of spectral sensing scheme is

$$
\frac{\partial \lambda_{\mathrm{N}}}{\partial n_{a}}=\frac{\frac{\partial\left(\Delta n_{\mathrm{eff}}\right)}{\partial n_{a}}}{\frac{1}{2 \cdot N \cdot L_{c}}-\frac{\partial\left(\Delta n_{\mathrm{eff}}\right)}{\partial \lambda_{\mathrm{N}}}} .
$$

\section{CONCLUSION}

In this paper, we have proposed two novel refractive index sensing schemes based on a solid twin-core microstructured optical fiber with a single microfluidic analyte channel centered between the two photonic bandgap guiding cores. From (4) and (6), the sensitivities of both the transmittance and spectral sensing schemes are proportional to the change in difference of supermode effective indices with analyte refractive index, $\partial\left(\Delta n_{\text {eff }}\right) / \partial n_{a}$.

For the transmittance sensing scheme the sensitivity to changes in analyte index (given by (4)) is maximized when $\Delta n_{\text {eff }}$ is small, i.e., at the short wavelength edge of the bandgap, and scales proportionally with sensor length. An example was given in which the transmittance of a $1 \mathrm{~mm}$ long sensor changed with analyte index by $65873 \% / \mathrm{RIU}$.

For the spectral sensing scheme, the sensitivity [given by (6)] is maximized when $\Delta n_{\mathrm{eff}}$ is independent of wavelength, i.e., at the long wavelength edge of the bandgap, and increases with the number of coupling lengths. An example was given of a sensor one coupling length long, in this case $334 \mu \mathrm{m}$, in which a change in analyte refractive index by 0.001 RIU led to a shift of coupling wavelength of $70 \mathrm{~nm}$, i.e., $70000 \mathrm{~nm} / \mathrm{RIU}$.

Theoretically, similar sensing characteristics can be achieved in all bandgaps of twin-core PBG MOFs, and such structures should be capable of measuring refractive index below the index of the fiber host material. The sensitivity and dynamic range of the proposed twin-core PBG MOF depend strongly on the photonic crystal structure, thus appropriate choice of the pitch $(\Lambda)$, diameter of rod $(d)$, material refractive indices, index contrast, and the diameter of the analyte channel can tailor and further optimize the performance of the proposed refractive index sensor.

\section{ACKNOWLEDGMENT}

W. Yuan thanks J. Lægsgaard, Y. Chen, and M. Yan for helpful discussions.

\section{REFERENCES}

[1] M. E. Bosch, A. J. R. Sánchez, F. S. Rojas, and C. B. Ojeda, "Recent development in optical fiber biosensors," Sensors, vol. 70, pp. 797-859, 2007.

[2] L. Rindorf, P. E. Hoiby, J. B. Jensen, L. H. Pedersen, O. Bang, and O. Geschke, "Towards biochips using microstructured optical fiber sensors," Anal. Bioanal. Chem., vol. 385, pp. 1370-1375, 2006.

[3] X. Fan, I. M. White, S. I. Shopova, H. Zhu, J. D. Suter, and Y. Sun, "Sensitive optical biosensors for unlabeled targets: A review," Anal. Chimica Acta, vol. 620, pp. 8-26, 2008

[4] RAPTOR - Portable Multianalyte Bioassay Detection System Research Int., Inc., Jun. 18, 2008. [Online]. Available: http://www.resrchintl.com/raptor-detection-system.html

[5] L. Rindorf, J. B. Jensen, M. Dufva, L. H. Pedersen, P. E. Høiby, and O. Bang, "Photonic crystal fiber long-period gratings for biochemical sensing," Opt. Expr., vol. 14, pp. 8224-8231, 2006. 
[6] J. R. Ott, M. Heuck, C. Agger, P. D. Rasmussen, and O. Bang, "Labelfree and selective nonlinear fiber-optical biosensing," Opt. Expr., vol. 16, pp. 20834-20847, 2008.

[7] L. Rindorf and O. Bang, "Highly sensitive refractometer with a photonic-crystal-fiber long-period grating," Opt. Lett., vol. 33, pp. 563-565, 2008.

[8] L. Rindorf and O. Bang, "Sensitivity of photonic crystal fiber grating sensors: Biosensing, refractive index, strain, and temperature sensing," J. Opt. Soc. Amer. B, vol. 25, pp. 310-324, 2008.

[9] D. K. C. Wu, B. T. Kuhlmey, and B. J. Eggleton, "Ultrasensitive photonic crystal fiber refractive index sensor," Opt. Lett., vol. 34, pp. 322-324, 2009.

[10] P. S. J. Russell, "Photonic crystal fibers," Science, vol. 299, pp. 358-362, 2003.

[11] P. S. J. Russell, "Photonic-crystal fibers," J. Lightw. Technol., vol. 24, pp. 4729-4749, 2006.

[12] R. F. Cregan, B. J. Mangan, J. C. Knight, T. A. Birks, P. S. J. Russell, P. J. Roberts, and D. C. Allan, "Single-mode photonic bandgap guidance of light in air," Science, vol. 285, pp. 1537-1539, 1999.

[13] D. Passaro, M. Foroni, F. Poli, A. Cucinotta, S. Selleri, J. Lægsgaard, and A. O. Bjarklev, "All-silica hollow-core microstructured Bragg fibers for biosensor application," IEEE Sensors J., vol. 8, no. 7, pp. 1280-1286, Jul. 2008.

[14] F. Luan, A. K. George, T. D. Hedley, G. J. Pearce, D. M. Bird, J. C. Knight, and P. S. J. Russell, "All-solid photonic bandgap fibers," Opt. Lett., vol. 29, pp. 2369-2371, 2004.

[15] G. Bouwmans, L. Bigot, Y. Quiquempois, F. Lopez, L. Provino, and M. Douay, "Fabrication and characterization of an all-solid 2D photonic bandgap fiber with a low-loss region $(<20 \mathrm{~dB} / \mathrm{km})$ around $1550 \mathrm{~nm}$," Opt. Expr., vol. 13, pp. 8452-8459, 2005.

[16] J. C. Knight, F. Luan, G. J. Pearce, A. Wang, T. A. Birks, and D. M. Bird, "Solid photonic bandgap fibers and applications," Jpn. J. Appl. Phys., vol. 45, pt. 1, pp. 6059-6063, 2006.

[17] G. Bouwmans, V. Pureur, A. Betourne, Y. Quiquempois, M. Perrin, L. Bigot, and M. Douay, "Progress in solid core photonic bandgap fibers," Opt. Quant. Electron., vol. 39, pp. 949-961, 2007.

[18] T. M. Monro, D. J. Richardson, N. G. R. Broderick, and P. J. Bennett, "Developing holey fibers for evanescent field devices," Electron. Lett., vol. 35, pp. 1188-1189, 1999.

[19] T. M. Monro, W. Belardi, K. Furusawa, J. C. Baggett, N. G. R. Broderick, and D. J. Richardson, "Sensing with microstructured optical fibers," Meas. Sci. Technol., vol. 12, pp. 854-858, 2001.

[20] J. M. Fini, "Microstructure fibers for optical sensing in gases and liquids," Meas. Sci. Technol., vol. 15, pp. 1120-1128, 2004.

[21] T. Ritari, J. Tuominen, H. Ludvigsen, J. C. Petersen, H. Sorensen, T. P. Hansen, and H. R. Simonsen, "Gas sensing using air-guiding photonic crystal fibers," Opt. Expr., vol. 17, pp. 4080-4087, 2004.

[22] B. J. Mangan, "Experimental study of dual-core photonic crystal fiber," Electron. Lett., vol. 36, pp. 1358-1359, 2000.

[23] F. Fogli, L. Accomandi, P. Bassi, G. Bellanca, and S. Trillo, "Full vectorial BPM modeling of index-guiding photonic crystal fibers and couplers," Opt. Expr., vol. 10, pp. 54-59, 2002.

[24] M. Hansen and G. E. Town, "Properties of dual-core couplers in microstructured optical fibers," in Proc. 28th Eur. Conf. Optical Commun., 2003, pp. 616-617.

[25] K. Saitoh, Y. Sato, and M. Koshiba, "Coupling characteristics of dual-core photonic crystal fiber couplers," Opt. Expr., vol. 11, pp. 3188-3195, 2003

[26] M. Chen and R. Yu, "Coupling characteristics of dual-core rectangular lattice photonic crystal fibers," J. Opt. A: Pure Appl. Opt., vol. 6, pp. 805-808, 2004

[27] J. Lægsgaard, O. Bang, and A. Bjarklev, "Photonic crystal fiber design for broadband directional coupling," Opt. Lett., vol. 29, pp. 2473-2475, 2004.

[28] J. Lægsgaard, "Directional coupling in twin-core photonic bandgap fibers," Opt. Lett., vol. 30, pp. 3281-3283, 2005.

[29] K. Reichenbach and C. Xu, "Independent core propagation in two-core photonic crystal fibers resulting from structural nonuniformities," Opt. Expr., vol. 13, pp. 10336-10348, 2005.

[30] K. Saitoh, N. J. Florous, M. Koshiba, and M. Skorobogatiy, "Design of narrow band-pass filters based on the resonant-tunneling phenomenon in multicore photonic crystal fibers," Opt. Expr., vol. 13, pp. 10327-10335, 2005.

[31] Z. Wang, T. Taru, T. A. Birks, and J. C. Knight, "Coupling in dual-core photonic bandgap fibers: Theory and experiment," Opt. Expr., vol. 15, pp. 4795-4803, 2007.

[32] M. Chen, Y. Zhang, and R. Yu, "Wavelength-selective coupling of dual-core photonic crystal fiber and its application," Chin. Opt. Lett., vol. 7, pp. 390-392, 2009.
[33] G. E. Town, R. F. Copperwhite, R. Kribich, K. O'Dwyer, and B. D. MacCraith, "Comparison of multimode and multichannel couplers for evanescent sensing of refractive index," in Proc. 30th Australian Conf. Optical Fiber Technol., Sydney, Australia, 2005.

[34] R. F. Copperwhite, M. Oubaha, G. E. Town, R. Kribich, K. O'Dwyer, and B. D. MacCraith, "Characterization of novel refractometric sensing systems," in Proc. SPIE Advanced Environmental, Chemical, and Biological Sensing Technologies III, Boston, MA, 2005, vol. 5993, pp. 78-88.

[35] M. A. van Eijkelenborg, M. C. J. Large, A. Argyros, J. Zagari, S. Manos, N. A. Issa, I. Bassett, S. Fleming, R. C. McPhedran, C. Martijn de Sterke, and N. A. P. Nicorovici, "Microstructured polymer optical fiber," Opt. Expr., vol. 9, pp. 319-327, 2001.

[36] M. C. J. Large, S. Ponrathnam, A. Argyros, I. M. Bassett, N. S. Pujari, F. Cox, R. Lwin, G. W. Barton, and M. A. van Eijkelenborg, "Microstructured polymer optical fibers: New opportunities and challenges," Mol. Cryst. Liq. Cryst., vol. 446, pp. 219-231, 2006.

[37] A. Argyros, M. A. van Eijkelenborg, M. C. J. Large, and I. M. Bassett, "Hollow-core microstructured polymer optical fiber," Opt. Lett., vol. 31, pp. 172-174, 2006.

[38] J. B. Jensen, P. E. Hoiby, G. Emiliyanov, O. Bang, L. H. Pedersen, and A. Bjarklev, "Selective detection of antibodies in microstructured polymer optical fibers," Opt. Expr., vol. 13, pp. 5883-5889, 2005.

[39] F. M. Cox, A. Argyros, and M. C. J. Large, "Liquid-filled hollow core microstructured polymer optical fiber," Opt. Expr., vol. 14, pp. 4135-4140, 2006.

[40] G. Emiliyanov, J. B. Jensen, O. Bang, P. E. Hoiby, L. H. Pedersen, E. M. Kjær, and L. Lindvold, "Localized biosensing with Topas microstructured polymer optical fiber," Opt. Lett., vol. 32, pp. 460-462, 2007.

[41] G. Emiliyanov, J. B. Jensen, O. Bang, P. E. Hoiby, L. H. Pedersen, E. M. Kjær, and L. Lindvold, "Localized biosensing with Topas microstructured polymer optical fiber: Erratum," Opt. Lett., vol. 32, p. 1059, 2007.

[42] K. Nielsen, H. K. Rasmussen, A. J. L. Adam, P. C. M. Planken, O. Bang, and P. U. Jepsen, "Bendable, low-loss Topas fibers for the terahertz frequency range," Opt. Expr., vol. 17, pp. 8592-8601, 2009.

[43] B. T. Kuhlmey, S. Coen, and S. Mahmoodian, "Coated photonic bandgap fibers for low-index sensing applications: Cut-off analysis," Opt. Express, vol. 17, pp. 16306-16321, 2009.

[44] S. G. Johnson and J. D. Joannopoulos, "Block-iterative frequency-domain methods for Maxwell's equations in a planewave basis," Opt. Expr., vol. 8, pp. 173-190, 2001.

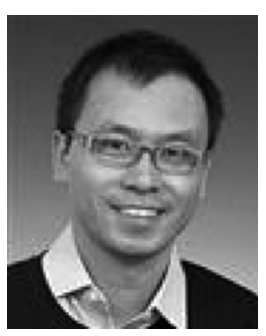

Wu Yuan received the $\mathrm{Ph} . \mathrm{D}$. degree in electronic engineering from the Chinese University of Hong Kong in September 2008.

He is a Postdoctoral Fellow with DTU Fotonik, Department of Photonics Engineering, Technical University of Denmark. His current research interests are in the field of fiber optics, including photonic crystal fibers, microstructured polymer optical fibers, fiber Bragg grating, fiber sensors, and biosensors.



Graham E. Town (M'83-SM'05) received the Bachelor of Engineering (Hons I) degree in electrical engineering from the NSW Institute of Technology (now the University of Technology), Sydney, Australia, in 1984 and the Doctor of Philosophy degree from the University of Sydney, Sydney, in 1992.

From 1991 to 2002, he was an academic in the Department of Electrical Engineering, University of Sydney, where he conducted research in the areas of magnetic resonance imaging, and subsequently in optical fiber lasers and photonics. He is currently a Professor of Electronic Engineering at Macquarie University, Sydney, where he initiated and developed the university's undergraduate engineering program. His current research interests are in fiber lasers and in guided-wave optical devices and their applications in telecommunication and sensing.

Dr. Town is a member of the Australian Optical Society, SPIE, and Engineers Australia. He has served as Chairperson of the NSW Joint Chapter of IEEE MTT/APS, was Founding Chairperson of the NSW Chapter of IEEE LEOS, and is currently Chairperson of the NSW Joint Chapter of IEEE Photonics/SSC/ CAS. 


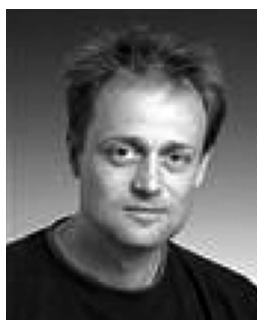

Ole Bang received the Master of Science degree in electrical engineering from the Technical University of Denmark in 1992 and the Doctor of Philosophy degree in nonlinear physics from the Technical University of Denmark, in 1993.

From 1993 to 1995, he was a Postdoctoral Fellow at the Laboratoire de Physique, Ecole Normale Supérieure de Lyon, France, where he worked on discrete physical models of the nonlinear dynamics of biomolecules. From 1995 to 1999, he was a Research Fellow at the Optical Sciences Centre, Australian National University, Canberra, Australia, where he worked theoretically on nonlinear optics, in particular on solitons and modulational instability in materials with a quadratic nonlinearity and quasi-phase-matching gratings. Since 1999, he has been an Associate Professor with the Technical University of Denmark, first at the Department of Informatics and Mathematical Modelling and, since 2003, with DTU Fotonik, Department of Photonics Engineering. His current research interests include microstructured optical fibers (MOFs) in silica and polymer, fiber-optical sensors for sensing biomolecules, stress, sound, and refractive index. He is also working on fabricating Bragg gratings, long-period gratings, and couplers in MOFs. Another main interest is supercontinuum generation and nonlinear fiber-optics in MOFs, as well as general nonlinear optics in nonlocal materials.

Dr. Bang is a member of the Optical Society of America and the Danish Optical Society. 